Ann. Zootech., I964, (4), 389-39r.

(NOTE PRÉLIMINAIRE)

\title{
RELATIONS ENTRE L'HYPERTROPHIE MUSCULAIRF. DES BOVINS CULARDS ET LE MÉTABOLISME DU COLLAGENE
}

\author{
R. BOCCARD \\ Avec la collaboration technique de Jeanne Fléchet \\ Laboratoire de Recherches sur la Viande \\ Centre national de Recherches zootechniques, Jouy-en-Josas (Seine-et-Oise)
}

Des manifestations typiques d'hypertrophie musculaire chez les bovins ont été décrites dans différentes races et dans divers pays (animaux " a groppa doppia" en Italie, "doppellender " en Allemagne, "double muscled ", en Grande-Bretagne et aux Etats-Unis, et " mulot " ou " cul de poulain ", ou "culard" en France) (LAUVERGNE et al., I963). On admet qu'il s'agit d'anomalies héréditaires, bien que les études génétiques menées depuis 50 ans n'aient pas pu établir définitivement si le caractère est lié à l'action d'un ou plusieurs couples de gènes, l'hypothèse d'un seul gène à dominance incomplète semble cependant prévaloir.

Les manifestations phénotypiques de ce caractère héréditaire sont multiples. Outre l'aspect général qui se traduit par des signes d'hypertrophie musculaire; les animaux présentent souvent des déformations osseuses ou des défauts dans les articulations. Par ailleurs, ils ont une peau plus fine : ainsi, pour des sujets de $500 \mathrm{~kg}$ (4 bovins normaux et 4 hypertrophiés), nous avons noté que les cuirs verts pesaient en moyenne respectivement 44 et $36 \mathrm{~kg}$. I'un autre côté,l'étude pondérale de l'organisation musculaire de 4 animaux hypertrophiés et de 4 témoins nous a révélé que l'hypertrophie affectait essentiellement les muscles superficiels, ce qui est en accord avec l'observation de LAWRIE, POMERoy et WiLiliams (I964).

Soumis à l'analyse chimique, 5 muscles de chacun des 8 animaux ont révélé une composition différente. Par rapport à la matière sèche, les muscles des animaux hypertrophiés ont moins de collagène et d'élastine insoluble, soit, en moyenne, respectivement, pour chacun des muscles : $3,7,4,5,4,6,5,6,6,4 \mathrm{p}$. Ioo contre 5,5, $6,2,5,7,8,9$, et $9, \mathrm{I}$ p. Ioo chez les animaux normaux, dont la teneur en muscles est plus faible. Ces résultats confirment ceux de L,AWrie, POMERoy et Wilírams (ig64) qui ont constaté de plus que la teneur en hydroxyproline de I4 muscles d'un animal normal était de I, I5 à 3,45 fois plus élevée que celle des muscles homologues de 
1'animal hypertrophié. Finfin, l'étude comparative de la tendreté des muscles montre que les muscles provenant d'animaux hypertrophiés sont en général plus tendres que les mêmes muscles des animaux normaux (DUMONT, Ig64).

L'ensemble de ces manifestations révèle des différences dans la nature ou l'importance des tissus conjonctifs.

L'exploration du métabolisme du collagène peut se faire indirectement par l'examen de l'excrétion urinaire de l'hydroxyproline (NEUBERGER et Richards, Ig64).

Sur 5 taurillons Charolais de 20 mois, reconnus comme présentant les signes de l'hypertrophie musculaire et sur 5 taurillons normaux, de même race et de même âge, nous avons suivi pendant 3 jours consécutifs, avant leur abattage, l'excrétion urinaire de l'azote total (méthode de Kjeldahl) et de 1'hydroxyproline (méthode Neuman et Logan).

L'excrétion d'azote total se révèle assez variable d'individu à individu, bien que les animaux aient sensiblement consommé durant les dernières semaines précédant leur abattage les mêmes quantités d'azote par $\mathrm{kg}$ de poids vif. Durant cette période, les croissances étaient en moyenne légèrement plus élevées pour les animaux normaux.

Dans l'urine des animaux "culards", le rapport de l'hydroxyproline à l'azote total est plus élevé que dans celle dans des animaux normaux, soit, en moyenne 0, I 302 contre 0,0996 , conformèrent aux résultats individuels suivants :

\begin{tabular}{cc} 
Bovins normaux & Bovins " culards" \\
\hline 0,094 & - \\
0,085 & $0, \mathrm{I} 54$ \\
$0, \mathrm{I05}$ & $0, \mathrm{I} 50$ \\
$0, \mathrm{II} 9$ & $0, \mathrm{I} 30$ \\
0,095 & $0, \mathrm{I} 04$ \\
\hline 0,0996 & $0, \mathrm{II} 3$ \\
\hline
\end{tabular}

Cette excrétion urinaire pourrait être, selon nous, le reflet de perturbations dans le métabolisme du collagène, 1'hydroxyproline libre n'étant pas incorporable dans la chaîne peptidique. Ces phénomènes auraient pour effet d'entraîner un déficit de la formation du collagène, ou une accélération de sa dégradation.

En conséquence, on peut imaginer qu'une grande partie des phénomènes phénotypiques de 1'hypertrophie musculaire peuvent relever de ces perturbations. En particulier, l'hypertrophie musculaire périphérique pourrait s'expliquer par l'hypotension résultant d'un défaut de conjonctif, tant dans la peau qu'à la périphérie des muscles eux-mêmes, par un phénomène semblable à celui mis en évidence par CRAWFORT (I954) qui a obtenu chirurgicalement chez le lapin une hypertrophie du tibialis anterior par la diminution de la tension de son tendon distal.

L'hypertrophie musculaire des bovins se distingue, par ailleurs, de celle obtenue par hypertension et qui entraîne une augmentation, tant en valeur relative qu'en valeur absolue, de tous les éléments protéiques constituant le muscle (VEcchionI et TARTARINI, I960).

I'hypothèse de perturbations du métabolisme du collagène chez les animaux 
hypertrophiés sera vérifiée sur un plus grand nombre d'animaux de types morphologiques variés ayant présenté des gains journaliers identiques et considérés à divers stades de leur croissance.

Dans la pratique, la différence d'excrétion urinaire de l'hydroxypronne pourrait éventuellement servir de test exploratoire de 1'amplitude de l'hypertrophie musculaire des bovins, et être une technique pour les études du déterminisme génétique de ce caractère.

Reçu pour publication en décembre 1964 .

\section{REMERCIEMENTS}

Je remercie B. L. Dumont, G. Fauconneau pour l'aide et les critiques apportées dans la discussion de cette hypothèse, et C. M. BÉRANGER pour la fourniture des échantillons.

\section{SUMMARY}

RELATIONS BETWEEN HEREDITARY MUSCULAR HYPERTROPHY IN CATTLE AND COLLAGEN METABOLISM (PRELIMINARY NOTE)

A hypothesis on the biochemical origin of muscular hypertrophy in cattle is given after consideration of the various associated symptoms.

It appears that in 2 groups of 5 animals, the ratio hydroxyproline to total $\mathrm{N}$ in urine is higher for hypertrophied than normal animals : $(0,1302$ versus 0,0996$)$.

\section{RÉFÉRENCES BIBLIOGRAPHIQUES}

Crawford C. N. C., r95t. An experimental study of muscle growth in the rabbit. J. Bone Jt. Surg., 36 B 294-303.

Dumont B. L., Ig64. Travaux en cours.

Necberger A., Richards F. F., I964. P'rotein biosynthesis in mammalian tissues. Part. II. Studies on turn over in the whole animal. In Munko H. N., Alussos J. B. Mammalian protein melabolism, vol. I, 1). 27 , Acad. Press., New York, London.

Lauvergie J. J., Vissac B., l'krramon A., 1963. Étude du caractère culard. I. Nise au point bibliographique. Amn. Loolech., 12, I33-156.

LAWrie R. A., Poyeroy R. W., Willians D. R., 1964 . Studies in the muscles of meat animals IV. Comparative composition of muscles from "doppelender " and normal sibling heifers. J. Agric. Sci, 62, 89-92.

Vecchioni R., Tartarini A., 1960. Comportamento delle frazioni collagene nella ipertrofia muscolare. Boll. Soc. Ilal. Biol. Sper., 26, I 4' 5-1417. 